TRANSACTIONS OF THE

AMERICAN MATHEMATICAL SOCIETY

Volume 359, Number 9, September 2007, Pages 4125-4142

S 0002-9947(07)04309-7

Article electronically published on April 11, 2007

\title{
A CRITERION FOR THE EQUIVALENCE OF THE BIRKHOFF-ROTT AND EULER DESCRIPTIONS OF VORTEX SHEET EVOLUTION
}

\author{
MILTON C. LOPES FILHO, HELENA J. NUSSENZVEIG LOPES, \\ AND STEVEN SCHOCHET
}

\begin{abstract}
In this article we consider the evolution of vortex sheets in the plane both as a weak solution of the two dimensional incompressible Euler equations and as a (weak) solution of the Birkhoff-Rott equations. We begin by discussing the classical Birkhoff-Rott equations with respect to arbitrary parametrizations of the sheet. We introduce a notion of weak solution to the Birkhoff-Rott system, and we prove consistency of this notion with the classical formulation of the equations. Our main purpose in this paper is to present a sharp criterion for the equivalence of the weak Euler and weak Birkhoff-Rott descriptions of vortex sheet dynamics.
\end{abstract}

\section{INTRODUCTION}

There are two distinct points of view in the mathematical description of interface dynamics. The more natural one is to explicitly propagate the interface itself using a time-dependent parametrization. An alternative approach is to embed the interface into the solution of a partial differential equation which can be evolved, carrying the interface with it. We will refer to the former as the explicit approach to interface dynamics, while the latter will be called the implicit approach. See 32] for a broad discussion and several instances of this dychotomy.

Vortex sheet evolution in two-dimensional, incompressible, ideal fluid flow is a classical example of interface dynamics for which both points of view have been widely addressed. The explicit approach in this context makes use of the BirkhoffRott equations. This system was originally derived by G. D. Birkhoff [3, and it is implicit in the work of N. Rott [35]. For the implicit point of view one uses the incompressible 2D Euler equations, regarding the vortex sheet as a feature of a suitably defined weak solution. The purpose of the present work is to establish a sharp smoothness criterion for the equivalence of these descriptions of vortex sheet motion.

The physically meaningful notion of generalized solution for the Euler equations is the weak form of the momentum equations for velocity and pressure, known as the weak velocity formulation. Indeed, the weak velocity formulation is an integral form of conservation of momentum, and therefore, it is close to physical first principles. In the problem of vortex sheet evolution, an alternative weak formulation, known as

Received by the editors March 4, 2005.

2000 Mathematics Subject Classification. Primary 76B03; Secondary 35Q35, 76B47.

Key words and phrases. Vortex sheets, incompressible flow, ideal flow, weak solutions.

(C)2007 American Mathematical Society 
the weak vorticity formulation, has proven to be more tractable. The weak vorticity formulation has been shown to be equivalent to the weak velocity formulation in all situations under consideration in this work; see [7, 37. In this context, it is natural to ask whether the Birkhoff-Rott description of vortex sheet evolution is equivalent to these weak formulations as well.

The problem of equivalence between Birkhoff-Rott and the weak forms of 2D Euler may be regarded as solved in the case of vortex sheets with smooth densities on smooth curves. Although there is no complete proof available, the proof of Theorem 6.1.2 in [27] (which assumes that the vortex sheet is a graph) can be easily adapted to establish such a result in general. On the other hand, it was recently shown, see 21, that a well-known exact solution of the Birkhoff-Rott equations fails to satisfy both the weak vorticity and the weak velocity formulations. Our main result is to show that, if the vortex sheet is a regular curve, for each fixed time, then the condition for equivalence is that the vorticity density be square-integrable with respect to arclength. The example in [21] shows that the integrability condition on the vorticity density is sharp. The definition of regular curve is standard in harmonic analysis, see [6]; a rectifiable curve is regular if its intersection with a ball of radius $r$ has length $\mathcal{O}(r)$.

In this work, we require a weak formulation of the Birkhoff-Rott equations. This is not a standard topic, and therefore, we must treat it at length. A special case of the weak Birkhoff-Rott equations formulated here was given in equation (6.1.14) of 27] for vortex sheets which are graphs. Furthermore, the argument used in 27] to establish equivalence between Birkhoff-Rott and 2D Euler in the smooth case should, in principle, be extendable to the weak solutions considered here. The method of proof we use is completely different. To motivate our particular weak formulation of the Birkhoff-Rott equations we have stated and proved a consistency result, namely, that the weak formulation of Birkhoff-Rott plus minimal regularity assumptions is equivalent to classical Birkhoff-Rott.

In order to properly contextualize our results we will give a brief account of the literature on vortex sheet evolution. Vortex sheets are a classical topic in fluid dynamics. The complicated evolution of vortex sheets is a natural source for the spontaneous appearance of small scale motion in incompressible fluids, an observation dating back to H. Helmholtz in 1868 (see the discussion and references in [1]). This motivates the continuing interest of the topic. The source of the small scales can be identified with a feature of vortex sheet motion, known as KelvinHelmholtz instability, see [36] and the references therein.

In the classical paper 29] D. Moore presented a theoretical account of how the Kelvin-Helmholtz instability could drive a real analytic vortex sheet to form curvature singularities in finite time. This work was later made rigorous by a number of authors, including results on existence of a local-in-time solution to Birkhoff-Rott for analytic initial data, 4, 11, 39] and formation of Moore's singularity [5, 11]. After singularity formation one expects vortex sheet roll-up. Numerical studies, see for example [17, illustrate the expected presence of double-branched spiral vortex sheets after singularity formation for periodic perturbations of a planar sheet. One is naturally led to study self-similar spiral shaped vortex sheets. The existence of exact self-similar spiral solutions of the Birkhoff-Rott equations goes back to an example due to L.Prandtl, generalized by T. Kambe; see [15, 33. These are examples of finite length logarithmic spirals. However, the generic roll-up after Moore's 
singularity and the roll-up of the elliptically loaded wing (another classical example) [16, 17, 18, 28, seem to lead to infinite length algebraic spirals for which no rigorous existence is known. An asymptotic description of self-similar solutions of the Birkhoff-Rott equations with algebraic spirals was first presented by Kaden [14] and generalized by Pullin 34. The Birkhoff-Rott equations are elliptic in nature, and there is a strong analogy between the Kelvin-Helmholtz instability and the Hadamard instability of the Cauchy problem for Laplace's equation. In particular, the explicit approach to the vortex sheet evolution problem is rather ill posed. Rigorous results in this direction have recently been presented by G. Lebeau and also by $\mathrm{S}$. Wu; see [19, 40.

The implicit approach to vortex sheets was pioneered by R. DiPerna and A. Majda in a series of papers [8, 9, 10], where they outlined a program for proving existence of weak solutions for the incompressible 2D Euler equations with vortex sheets as initial data. The DiPerna-Majda program was carried out in the case of steady weak solutions but remains open in general [12, 24, 31, 41]. In 1990, J.M. Delort proved the existence of a global-in-time weak solution for the vortex sheet initial data problem with distinguished sign [7]; see also [13, 20, 25, 37, 38. A globalin-time existence result has also been proved in the case of mirror-symmetric flows with distinguished sign vorticity on each side of the mirror [23]. Delort's Theorem and its mirror-symmetric extension provide the existence of a meaningful evolution for certain vortex sheets beyond singularity formation but give no information on their structure.

The remainder of this article is divided into three sections. In the next section we describe various formulations of both the Euler equations and the Birkhoff-Rott equations, and we discuss the consistency of the weak formulation of the latter. The following section contains the precise statement and proof of the equivalence between weak Birkhoff-Rott and the weak vorticity formulation of 2D Euler, as well as the discussion of the sharpness of this result. The final section contains some interpretations of the work presented, as well as open problems and conclusions. This work contains a complete answer to a problem formulated by $\mathrm{S}$. Wu at the end of [40].

\section{VorteX SheET EQUATIONS IN WEAK FORM}

This section is divided in three subsections. In the first we recall the weak vorticity formulation of the 2D incompressible Euler equations, implementing it in the special case where vorticity is concentrated on a curve. In the second subsection we discuss the derivation of the several forms of the Birkhoff-Rott system. In the third we introduce a notion of weak solution of the Birkhoff-Rott equations, and we prove consistency of this notion with the classical form of the equations.

2.1. Weak forms for the vorticity equation. The vorticity formulation of the $2 \mathrm{D}$ incompressible Euler equations is

$$
\begin{aligned}
\omega_{t}+u \cdot \nabla \omega & =0, \\
u & =K * \omega, \\
\omega(x, 0) & =\omega_{0}(x),
\end{aligned}
$$


with

$$
K(x)=\frac{x^{\perp}}{2 \pi|x|^{2}},
$$

where $\left(x_{1}, x_{2}\right)^{\perp}=\left(-x_{2}, x_{1}\right), \omega$ is the vorticity, $u=\left(u_{1}, u_{2}\right)$ is the velocity and $K=\left(K_{1}, K_{2}\right)$ is the kernel of the Biot-Savart law. This system of equations can be reformulated in several different ways that are relevant for comparison with the Birkhoff-Rott equations. First, the Lagrangian representation is obtained by noting that (2.1) says that $\omega$ is preserved along the particle trajectories having velocity $u$, i.e.,

$$
\begin{aligned}
\omega\left(\Psi\left(x_{0}, t\right), t\right) & =\omega_{0}\left(x_{0}\right), \quad \text { where } \\
\frac{d}{d t} \Psi\left(x_{0}, t\right) & =u\left(\Psi\left(x_{0}, t\right), t\right), \\
\Psi\left(x_{0}, 0\right) & =x_{0},
\end{aligned}
$$

with $u$ defined as before by the Biot-Savart law (2.2).

Vortex sheet flows have vorticities which are Radon measures supported on rectifiable curves. In order to study such flows we require a weak formulation of the vorticity equation. There are actually two versions: The traditional weak vorticity formulation (e.g. [27]) is obtained by multiplying (2.1) by a smooth test function $\varphi$ having compact support in $[0, T) \times \mathbb{R}^{2}$, integrating over space and time, and integrating by parts, which yields

$$
\int_{0}^{T} \int_{\mathbb{R}^{2}} \omega(x, t)\left\{\varphi_{t}+u(x, t) \cdot \nabla \varphi\right\} d x d t+\int_{\mathbb{R}^{2}} \omega_{0}(x) \varphi(x, 0) d x=0,
$$

with $u$ still given by (2.2).

The velocity associated with a vortex sheet is, in principle, discontinuous on the sheet, a fact which we will discuss in great detail later. The discontinuity at the sheet implies that the term $\omega u \cdot \nabla \varphi$ appearing in (2.8) is not well defined. However, this difficulty can be overcome by considering an alternative weak formulation as follows.

The modern weak form can be obtained [37 from (2.8) by substituting $u$ by $K * \omega$, see (2.2), and replacing the factor multiplying $\omega(x, t) \omega(y, t)$ in the result by its symmetric part. This yields

$$
\begin{array}{r}
\int_{0}^{T} \int_{\mathbb{R}^{2}} \varphi_{t} \omega(x, t) d x d t+\int_{0}^{T} \int_{\mathbb{R}^{2}} \int_{\mathbb{R}^{2}} H_{\varphi}(x, y, t) \omega(y, t) \omega(x, t) d x d y d t \\
+\int_{\mathbb{R}^{2}} \varphi(x, 0) \omega_{0}(x) d x=0,
\end{array}
$$

where the function

$$
H_{\varphi}(x, y, t):=\frac{\nabla \varphi(x, t)-\nabla \varphi(y, t)}{2} \cdot K(x-y)
$$

is continuous for $x \neq y$, and it is also bounded.

Let $\mathcal{C B M}$ denote the space of bounded Radon measures with no discrete part.

Definition 2.1. Let $\omega \in L^{\infty}\left((0, T) ; \mathcal{C B M}\left(\mathbb{R}^{2}\right)\right)$. We will say that $\omega$ is a weak solution of the Euler equations with initial data $\omega_{0}$ if, for any test function $\varphi \in$ $C_{c}^{\infty}\left([0, T) \times \mathbb{R}^{2}\right),(2.9)$ holds. 
For smooth vorticities $\omega$ decaying sufficiently rapidly at infinity, all four formulations (2.1)-(2.3), (2.5) $-(2.7)$ plus (2.2), (2.8) plus (2.2), and (2.9) are equivalent [7. 27, 36, 37, to each other and to both the classical and weak [8] velocity formulations of the Euler equations. Moreover [7, 37, the modern weak formulation remains equivalent to the weak velocity formulation assuming only that the vorticity lies in $L^{\infty}([0, T], \mathcal{B M}) \cap L^{\infty}\left([0, T], H_{\text {loc }}^{-1}\right)$.

It was shown in 37. that the modern weak vorticity formulation makes sense whenever the vorticity is a bounded measure having no discrete part. In particular, it makes sense when the vorticity is a measure concentrated along a smooth timedependent curve.

Let $\mathcal{C}_{t}$ be a smooth, time-dependent curve,

$$
\mathcal{C}_{t}:=\left\{\xi=\xi(s, t) \mid s_{0} \leq s \leq s_{1}\right\},
$$

parametrized by arclength. Let $\gamma=\gamma(s, t)$ be a smooth density and specify the vorticity to be the measure $\omega=\omega(x, t)=\gamma \delta_{\mathcal{C}_{t}}$ defined through the identity

$$
\langle\omega(\cdot, t), \varphi\rangle \equiv \int_{s_{0}}^{s_{1}} \gamma(s, t) \varphi(\xi(s, t)) d s
$$

for any test function $\varphi \in C_{c}^{\infty}\left(\mathbb{R}^{2}\right)$. We assume that the initial vorticity is of the same form. If $\omega$ satisfies Definition 2.1, then we substitute (2.11) into (2.9) to get

$$
\begin{aligned}
& \int_{0}^{T} \int_{s_{0}(t)}^{s_{1}(t)} \varphi_{t}(\xi(s, t), t) \gamma(s, t) d s d t \\
& +\int_{0}^{T} \int_{s_{0}(t)}^{s_{1}(t)} \int_{s_{0}(t)}^{s_{1}(t)} H_{\varphi}(\xi(r, t), \xi(s, t), t) \gamma(r, t) \gamma(s, t) d r d s d t \\
& +\int_{s_{0}(0)}^{s_{1}(0)} \varphi\left(\xi_{0}(s), 0\right) \gamma_{0}(s) d s=0,
\end{aligned}
$$

for any test function $\varphi \in C_{c}^{\infty}\left([0, T) \times \mathbb{R}^{2}\right)$.

2.2. The Birkhoff-Rott system. We will now change our point of view, discussing the explicit approach to vortex sheet evolution. Our objective is to examine the equation for the evolution of the sheet with respect to an arbitrary parametrization. To this end let us begin by considering the linear problem of transport, by a smooth vector field, of a measure concentrated on a smooth curve in the plane.

Fix $v$ a given smooth vector field in the plane. Denote by $X=X_{t}$ the flow to time $t$ generated by $v$. Let $\mu_{0}$ be a Radon measure on the plane. We say that $\mu=\mu(\cdot, t)$ is the transport by $v$ of the measure $\mu_{0}$ if, for any Borelian subset $E \subseteq \mathbb{R}^{2}$, we have:

$$
\mu(E, t)=\mu_{0}\left(X_{-t}(E)\right) .
$$

It is not hard to see that, if $\mu_{0}=\gamma_{0} \delta_{\mathcal{C}_{0}}$, then the transport by $v$ is of the form $\mu(\cdot, t)=\gamma \delta_{\mathcal{C}_{t}}$ with $\mathcal{C}_{t}=X_{t}\left(\mathcal{C}_{0}\right)$. Furthermore, under the same hypothesis, if $\Sigma_{t} \subseteq \mathcal{C}_{t}$ is the transport of a portion $\Sigma_{0}$ of $\mathcal{C}_{0}$, then

$$
\frac{d}{d t} \int_{\Sigma_{t}} \gamma \cdot d \ell=0
$$


Lemma 2.1. Let $\mu_{0}=\gamma_{0} \delta_{\mathcal{C}_{0}}$ be a Radon measure with support on a smooth curve $\mathcal{C}_{0}$. Let $\mu=\mu(\cdot, t)$ be the transport by $v$ of the measure $\mu_{0}$.

Let $y=y(\eta, t)$ be a parametrization of $\mathcal{C}_{t}$ and denote

$$
\sigma(\eta, t):=\gamma(s(\eta, t), t) \frac{\partial s}{\partial \eta},
$$

where $s=s(\eta, t)$ is arclength with respect to a reference point.

Then there exists $a=a(\eta, t)$ such that the following equations are satisfied:

$$
\left\{\begin{array}{l}
y_{t}+a y_{\eta}=v \\
\sigma_{t}+(a \sigma)_{\eta}=0
\end{array}\right.
$$

Proof. Consider a parametrization of $\mathcal{C}_{0}, z_{0}=z_{0}(\theta), \theta \in I \subseteq \mathbb{R}$, and we assume for convenience that $0 \in I$. Let $z(\theta, t)=X_{t}\left(z_{0}(\theta)\right)$ be a (Lagrangian) parametrization of $\mathcal{C}_{t}$. Let $s=s(\theta, t)$ be the arclength along $\mathcal{C}_{t}$ between $z(0, t)$ and $z(\theta, t)$. Then $\theta \mapsto s(\theta, t)$ is an invertible change-of-variables. We write $\xi=\xi(s, t)$ for the parametrization with respect to the arclength $s$, measured from $z(0, t)$.

With this notation it is a straightforward calculation to verify that

$$
\xi_{t}+\widetilde{a}(s, t) \xi_{s}=v,
$$

with

$$
\widetilde{a}(s, t)=\frac{\partial s}{\partial t}(\theta(s, t), t) .
$$

Next, implementing the condition (2.13) gives, for any $\theta_{0}, \theta_{1}$,

$$
\frac{d}{d t} \int_{s\left(\theta_{0}, t\right)}^{s\left(\theta_{1}, t\right)} \gamma(s, t) d s=0
$$

From this integral equation it follows easily that

$$
\gamma_{t}+(\widetilde{a}(s, t) \gamma)_{s}=0 .
$$

If $y=y(\eta, t)$ is any other parametrization of $\mathcal{C}_{t}$ and if $\sigma=\gamma s_{\eta}$, then it is immediate that $y$ and $\sigma$ satisfy (2.14) with

$$
a(\eta, t)=\frac{\widetilde{a}(s(\eta, t), t)-s_{t}(\eta, t)}{s_{\eta}(\eta, t)} .
$$

This concludes the proof.

Remark. System (2.14) is an explicit description of the propagation of a curve which corresponds to the implicit description given by the equation $\mu_{t}+\operatorname{div}(v \mu)=0$, in the sense of distributions. Note that the function $a$ is a free parameter in (2.14), not a variable. Each choice of $a$ gives rise to a different parametrization of the evolution of the same time-dependent measure. System (2.14) shows that to propagate such a measure all we require is the propagating velocity field on the curve itself.

Now let us return to the vortex sheets themselves. We assume vorticity is of the form $\omega=\gamma \delta_{\mathcal{C}_{t}}$, and we parametrize $\mathcal{C}_{t}$ by a function $y=y(\eta, t)$, with $\sigma=\gamma d s / d \eta$, as before. The velocity associated to points $x$ outside the vortex sheet can be expressed by the Biot-Savart law (2.2):

$$
u(x, t)=\int_{\eta_{0}}^{\eta_{1}} K(x-y(\eta, t)) \sigma(\eta, t) d \eta,
$$

with $K$ given by (2.4), since $\sigma$ already includes the element of length of the curve. 
It is a well-known fact that the flow (2.15), induced by the vortex sheet, is discontinuous across the sheet. More precisely, the normal component of $u$ at $\mathcal{C}_{t}$ is continuous, whereas the tangential component has a jump discontinuity with magnitude given precisely by $\gamma$. These are nontrivial facts, and the reader may consult [36] for a thorough discussion.

Observe that the motion of the curve $\mathcal{C}_{t}$ is completely determined by the extension to $\mathcal{C}_{t}$ of the normal component of $u$. On the other hand, to propagate the density $\gamma$ in an explicit manner one needs to make a choice of tangential component of velocity on the sheet. This choice must take into account the nonlinear nature of the problem, in a way that we will explore later. For the present discussion, let us simply consider the standard choice, which is to prescribe the velocity of the sheet as the arithmetic mean of the limit velocity from each side of the sheet. This arithmetic mean, when calculated using the velocity defined by (2.15), can be expressed as a principal value integral in the following way:

$$
\begin{aligned}
& \mathcal{U}[y ; \sigma](\eta, t) \equiv p \cdot v \cdot \int_{a_{0}}^{a_{1}} K\left(y(\eta, t)-y\left(\eta^{\prime}, t\right)\right) \sigma\left(\eta^{\prime}, t\right) d \eta^{\prime} \\
= & \lim _{\varepsilon \rightarrow 0^{+}} \int_{\left|y(\eta, t)-y\left(\eta^{\prime}, t\right)\right| \geq \varepsilon} K\left(y(\eta, t)-y\left(\eta^{\prime}, t\right)\right) \sigma\left(\eta^{\prime}, t\right) d \eta^{\prime} ;
\end{aligned}
$$

see [36].

We use the vector field $\mathcal{U}$ to propagate $\omega=\gamma \delta_{\mathcal{C}_{t}}$. We assume that the evolution of $\omega$ can be described by a system of the form (2.14) with transporting velocity $v=\mathcal{U}$ as follows:

$$
\left\{\begin{array}{l}
y_{t}+a(\eta, t) y_{\eta}=\mathcal{U}[y ; \sigma] \\
\sigma_{t}+(a(\eta, t) \sigma)_{\eta}=0 .
\end{array}\right.
$$

System (2.17) is a general form of the classical Birkhoff-Rott system. One may close system (2.17) by prescribing $a$. For instance, if one assumes that the vortex sheet is the graph of a function of $x$, and parametrizes it using $x$ itself, then $a$ is the first component of $\mathcal{U}[y ; \sigma]$; see [27. The choice of a Lagrangian parametrization, i.e., such that $\partial y / \partial t=\mathcal{U}[y ; \sigma]$, corresponds to choosing $a=0$. The scalar $a$ measures how much the evolution of a chosen parametrization fails to be Lagrangian. The circulation parametrization $\Gamma=\Gamma(s, t) \equiv \int_{0}^{s} \gamma\left(s^{\prime}, t\right) d s^{\prime}$, with $s$ being arclength, is a special case of Lagrangian parametrization for which $\sigma \equiv 1$. It gives rise to the traditional form of the Birkhoff-Rott equations,

$$
\partial_{t} \bar{z}=\frac{1}{2 \pi} p \cdot v \cdot \int \frac{1}{z(\Gamma, t)-z\left(\Gamma^{\prime}, t\right)} d \Gamma^{\prime},
$$

where we have switched to complex variable notation for the position of the sheet.

In this work, we will choose to parametrize vortex sheets by arclength. In this case, the function $a$ must become another unknown and the Birkhoff-Rott equations become:

$$
\left\{\begin{array}{l}
\xi_{t}+a(s, t) \xi_{s}=\mathcal{U}[\xi ; \gamma] \\
\gamma_{t}+(a(s, t) \gamma)_{s}=0 \\
\left|\frac{\partial \xi}{\partial s}\right|=1
\end{array}\right.
$$

One may also fix the origin of the arclength parametrization by taking $a(0, t) \equiv 0$; see 40. 
Remark. How does one justify the use of the arithmetic mean in extending the Biot-Savart velocity to the vortex sheet? This is, in a sense, the key issue behind the present work. One could offer a convincing argument, approximating the evolution of the vortex sheet by desingularizing the Biot-Savart kernel, using Lemma 2.1 for this situation to get an approximate Birkhoff-Rott system and passing to the limit. This can be done rigorously if we assume that the approximate evolution is convergent and if we also show that natural desingularizations indeed lead to the principal value integral (2.16). A stronger version of the argument outlined above was carried out by Benedetto and Pulvirenti, who proved that the evolution of vortex sheets by Birkhoff-Rott is the asymptotic description of thin shear bands under the Euler equations; see 2. Another possibility is to argue that the arithmetic mean is the only extension that leads to vortex sheet evolution compatible with 2D Euler. This approach has been carried out in several manners; see [3, 26, 27, 36]. In all cases, smoothness of the vortex sheet and its density have been assumed. One of the motivations of the present work is to determine how irregular the vortex sheet can be, while retaining the compatibility of the choice of arithmetic mean in (2.14) with incompressible 2D Euler.

2.3. Weak form of the Birkhoff-Rott system. Our goal is to compare solutions of the Birkhoff-Rott equations and of the vorticity equation having limited smoothness. To do so, we require a weak formulation of the Birkhoff-Rott equations. Such a weak formulation can be obtained by formally substituting the vorticity (2.11) into the traditional weak formulation of the vorticity equation, given by (2.8) and replacing $u$ by $\mathcal{U}$. We use the traditional rather than the modern weak formulation (2.9) of the vorticity equation because, as we have discussed in the previous subsection, the hallmark of the Birkhoff-Rott equation is the choice of the arithmetic mean in extending velocity to the sheet, or equivalently, the introduction of the principal value in the integral defining the velocity. The issue is that the velocity does not appear in the modern weak formulation of the vorticity equation. Plugging (2.11) into (2.8) and replacing $u$ by $\mathcal{U}$ yields

$$
\begin{aligned}
\int_{0}^{T} \int_{s_{0}}^{s_{1}} \gamma(s, t)\left\{\varphi_{t}(\xi(s, t), t)+\mathcal{U}[\xi, \gamma](s, t)\right. & \cdot \nabla \varphi(\xi(s, t), t)\} d s d t \\
& +\int_{s_{0}}^{s_{1}} \gamma_{0}(s) \varphi\left(\xi_{0}(s), 0\right) d s=0 .
\end{aligned}
$$

Definition 2.2. Let $\mathcal{C}_{t}=\left\{\xi=\xi(s, t) \mid s_{0}(t) \leq s \leq s_{1}(t)\right\}$ be a rectifiable curve for each $t \in[0, T)$. Let $\gamma=\gamma(s, t) \in L^{\infty}\left((0, T) ; L^{1}(d s)\right)$ be such that $\mathcal{U}[\xi ; \gamma]$ is defined and $\gamma \mathcal{U}[\xi ; \gamma] \in L^{\infty}\left((0, T) ; L^{1}(d s)\right)$. We say that $(\gamma, \xi)$ is a weak solution of the Birkhoff-Rott equations with initial data $\left(\gamma_{0}, \xi_{0}\right)$ if (2.19) holds for every test function $\varphi \in C_{c}^{\infty}\left([0, T) \times \mathbb{R}^{2}\right)$.

Our next step is to prove that Definition 2.2 is compatible with the Birkhoff-Rott system (2.18). For vortex sheets of finite length we will need to supplement (2.18) by (moving) boundary conditions. It is natural to assume that the endpoints $s_{0}(t)$ and $s_{1}(t)$ of the curve are material trajectories, although this hypothesis will only be needed when the vorticity density is nonzero there. We will therefore assume that

$$
\gamma\left(s_{i}(t), t\right)\left[s_{i}^{\prime}(t)-a\left(s_{i}(t), t\right)=0\right], i=0,1
$$


Even when the vortex sheet has infinite length, if it is contained in a bounded region, then some form of boundary condition is still needed. It will suffice to assume in this case that

$$
\int_{s_{0}(t)}^{s_{1}(t)}|\gamma(s, t)| d s \leq c<\infty
$$

which is reasonable since it makes the mass of vorticity locally finite.

We now show that for smooth enough functions $\xi$ and $\gamma$, being a weak solution of the Birkhoff-Rott equations using arclength parametrization is equivalent to satisfying the classical Birkhoff-Rott system (2.18):

Theorem 2.2. Let $\xi=\xi(s, t), \gamma=\gamma(s, t)$ and $a=a(s, t)$ be solutions of (2.18) in $C_{b}^{1}(\Omega)$, where $\Omega:=\left\{(s, t) \mid s_{0}(t) \leq s \leq s_{1}(t), 0 \leq t<T\right\}$. Assume also that if an endpoint $s_{j}(t)$ is finite, then (2.20) is satisfied, while if $s_{j}(t)$ is infinite but $\xi\left(s_{j}(t), t\right)$ is finite, then (2.21) holds. Then $(\gamma, \xi)$ is a weak solution of the BirkhoffRott equations with initial data $(\gamma(s, 0), \xi(s, 0))$ in the sense of Definition 2.2.

Conversely, suppose that $(\gamma, \xi)$ is a weak solution of the Birkhoff-Rott equations with initial data $\left(\gamma_{0}, \xi_{0}\right)$ in the sense of Definition 2.2, where $s$ is an arclength parameter and at each fixed time $\xi$ is one-to-one except that $\xi\left(s_{1}(t), t\right)$ is allowed to equal $\xi\left(s_{0}(t), t\right)$. Assume in addition the following prescribed regularity:

(1) The parametrization $\xi$ and the density $\gamma$ are $C_{b}^{1}$ on $\Omega$.

(2) The velocity $\mathcal{U}[\xi ; \gamma]$ is $C_{b}^{0}$ on $\Omega$.

(3) If $\left|s_{i}\right|<\infty$, then $s_{i}$ is $C_{b}^{1}([0, T))$.

Then $\gamma$ and $\xi$ satisfy (2.18) and (2.20) with $a:=\xi_{s} \cdot\left[\mathcal{U}-\xi_{t}\right]$, except that the equation for $\xi$ need not hold in any open set on which $\gamma$ vanishes identically.

Remarks. (1) Suitably interpreted, Theorem 6.1 .1 of [27 shows a version of the first half of Theorem 2.2 for vortex sheets parametrized by one of the components of $x$. To see this, note that, as remarked on the next page there, the integral $\int \omega u$ is not well-defined on the curve but must be given meaning via a principal-value integral.

(2) The proviso that the evolution equation for $\xi$ need not hold where $\gamma$ vanishes is reasonable, since such regions are in essence not really part of the vortex sheet. Furthermore, the evolution equation for $\gamma$ implies that if it is nonzero everywhere on the sheet initially it will remain so at later times.

Proof. Let $\xi=\xi(s, t), \gamma=\gamma(s, t)$ and $a=a(s, t)$ be $C_{b}^{1}$ solutions of (2.18). In the following calculations we will assume that $\left(s_{0}(t), s_{1}(t)\right)$ is a bounded interval for each $0 \leq t<T$, but the case when either or both $s_{j}$ are infinite will also be treated. Let $\varphi$ belong to $C_{c}^{\infty}\left([0, T) \times \mathbb{R}^{2}\right)$. Multiplying the equation for $\gamma$ in (2.17) by $-\varphi(\xi(s, t), t)$, integrating over $s$ and $t$, and then integrating by parts yields

$$
\begin{aligned}
0= & -\int_{0}^{T} \int_{s_{0}(t)}^{s_{1}(t)}\left[\gamma_{t}+(a \gamma)_{s}\right] \varphi(\xi(s, t), t) d s d t \\
= & \int_{0}^{T} \int_{s_{0}(t)}^{s_{1}(t)} \gamma\left[\varphi_{t}+\xi_{t} \cdot \nabla \varphi+a \xi_{s} \cdot \nabla \varphi\right] d s d t \\
& +\int_{s_{0}(0)}^{s_{1}(0)} \gamma(s, 0) \varphi(\xi(s, 0), 0) d s+\left.\left.\int_{0}^{T} \gamma \varphi\right|_{s=s_{j}(t)}\left[s_{j}^{\prime}(t)-a\left(s_{j}(t), t\right)\right]\right|_{j=0} ^{j=1} d t .
\end{aligned}
$$


If $s_{j}(t)$ is finite, then the corresponding boundary term vanishes by (2.20). If $s_{j}(t)$ is infinite and $\xi\left(s_{j}(t), t\right)$ is also infinite, then that boundary term vanishes because $\varphi$ has compact support. If $s_{j}(t)$ is infinite but $\xi\left(s_{j}(t)\right)$ is finite, then the integrability of $\gamma$ combined with the boundedness of its derivative implies that $\gamma$ tends to zero as $s \rightarrow s_{j}$, so that the boundary term still vanishes. Upon taking into account the equation for $\xi$ in (2.17), (2.22) reduces to

$$
\begin{aligned}
0= & \int_{0}^{T} \int_{s_{0}(t)}^{s_{1}(t)} \gamma(s, t)\left[\varphi_{t}(\xi(s, t), t)+\mathcal{U}[\xi, \gamma](s, t) \cdot \nabla \varphi(\xi(s, t), t)\right] d s d t \\
& +\int_{s_{0}(0)}^{s_{1}(0)} \gamma(s, 0) \varphi(\xi(s, 0), 0) d s,
\end{aligned}
$$

which shows that (2.19) holds with $\gamma_{0}(s)=\gamma(s, 0)$ and $\xi_{0}(s)=\xi(s, 0)$. Since $\gamma$ lies in $L^{1}$ by assumption, and the conditions on $\xi$ and $a$ imply that $\mathcal{U}$ is bounded, the other conditions of Definition 2.2 are also satisfied.

Conversely, let $\gamma$ and $\xi$ be a weak solution of Birkhoff-Rott in the sense of Definition 2.2 satisfying the regularity assumptions in the statement. Again, we will assume in our computation that $s_{0}(t)$ and $s_{1}(t)$ are finite; if not, then the boundary terms vanish. Let $\varphi \in C_{c}^{\infty}\left([0, T) \times \mathbb{R}^{2}\right)$. Taking $\varphi(x, t)=e^{-t / \varepsilon} \psi(x)$ in (2.19), letting $\varepsilon \rightarrow 0$, and using the assumed regularity shows that $\xi_{0}(s)=\xi(s, 0)$ and $\gamma_{0}(s)=\gamma(s, 0)$. Integration by parts in (2.19) therefore yields

$$
\begin{aligned}
0= & \int_{0}^{T} \int_{s_{0}(t)}^{s_{1}(t)} \gamma(s, t)\left[\varphi_{t}(\xi(s, t), t)+\mathcal{U} \cdot \nabla \varphi(\xi(s, t), t)\right] d s d t \\
& +\int_{s_{0}(0)}^{s_{1}(0)} \gamma(s, 0) \varphi(\xi(s, 0), 0) d s \\
= & \int_{0}^{T} \int_{s_{0}(t)}^{s_{1}(t)} \gamma(s, t)\left[\frac{d}{d t} \varphi(\xi(s, t), t)+\left\{\mathcal{U}-\xi_{t}\right\} \cdot \nabla \varphi(\xi(s, t), t)\right] d s d t \\
& +\int_{s_{0}(0)}^{s_{1}(0)} \gamma(s, 0) \varphi(\xi(s, 0), 0) d s \\
= & \int_{0}^{T} \int_{s_{0}(t)}^{s_{1}(t)} \gamma\left\{\mathcal{U}-\xi_{t}\right\} \cdot \nabla \varphi(\xi(s, t), t)-\gamma_{t} \varphi(\xi(s, t), t) d s d t \\
& -\left.\left.\int_{0}^{T} \gamma \varphi\right|_{s=s_{j}(t)} s_{j}^{\prime}(t)\right|_{j=0} ^{j=1} d t .
\end{aligned}
$$

Since $\xi(s, t)$ is an arclength parametrization at each fixed time, $\left|\xi_{s}\right|=1$, and hence $\mathcal{U}-\xi_{t}=a(s, t) \xi_{s}+b(s, t) \xi_{s}^{\perp}$ with $a=\xi_{s} \cdot\left[\mathcal{U}-\xi_{t}\right]$ being continuous. Substituting this into (2.23) yields

$$
\begin{aligned}
0= & \int_{0}^{T} \int_{s_{0}(t)}^{s_{1}(t)}\left[a \gamma \partial_{s} \varphi(\xi(s, t), t)-\gamma_{t} \varphi(\xi(s, t), t)\right] d s d t \\
& +\int_{0}^{T} \int_{s_{0}(t)}^{s_{1}(t)} b \gamma \xi_{s}^{\perp} \cdot \nabla \varphi(\xi(s, t), t) d s d t-\left.\left.\int_{0}^{T} \gamma \varphi\right|_{s=s_{j}(t)} s_{j}^{\prime}(t)\right|_{j=0} ^{j=1} d t .
\end{aligned}
$$


Since no time derivatives are applied to $\varphi$ in (2.24), taking $\varphi=\eta_{\varepsilon}(t) \psi(x)$ with $\eta_{\varepsilon}(t) \rightarrow \delta\left(t-t_{0}\right)$ shows that for every $t \in(0, T)$,

$$
\begin{aligned}
0= & \int_{s_{0}(t)}^{s_{1}(t)}\left[a \gamma \partial_{s} \psi(\xi(s, t))-\gamma_{t} \psi(\xi(s, t))\right] d s \\
& +\int_{s_{0}(t)}^{s_{1}(t)} b \gamma \xi_{s}^{\perp} \cdot \nabla \psi(\xi(s, t)) d s-\left.\left.\gamma \psi\right|_{s=s_{j}(t)} s_{j}^{\prime}(t)\right|_{j=0} ^{j=1} .
\end{aligned}
$$

Now pick any $s_{*} \in\left(s_{0}(t), s_{1}(t)\right)$, and take

$$
\psi(x)=\xi_{s}^{\perp}\left(s_{*}, t\right) \cdot\left[x-\xi\left(s_{*}, t\right)\right] \frac{\eta\left(\frac{\left|x-\xi\left(s_{*}, t\right)\right|^{2}}{\varepsilon^{2}}\right)}{\varepsilon},
$$

where $\eta$ is an even $C_{c}^{\infty}$ function. Since

$$
\xi(s, t)-\xi\left(s_{*}, t\right)=\xi_{s}\left(s_{*}, t\right) \cdot\left(s-s_{*}\right)+o\left(s-s_{*}\right),
$$

we obtain the estimates $\left|\xi(s, t)-\xi\left(s_{*}, t\right)\right|=O\left(\left|s-s_{*}\right|\right)$,

$$
\begin{aligned}
\psi(\xi(s, t)) & =o\left(s-s_{*}\right) \frac{\eta\left(\frac{\left|x-\xi\left(s_{*}, t\right)\right|^{2}}{\varepsilon^{2}}\right)}{\varepsilon} \\
\xi_{s}^{\perp}\left(s_{*}, t\right) \cdot \nabla \psi(\xi(s, t)) & =\frac{\eta\left(\frac{\left|x-\xi\left(s_{*}, t\right)\right|^{2}}{\varepsilon^{2}}\right)}{\varepsilon}+\frac{o\left(\left(s-s_{*}\right)^{2}\right)}{\varepsilon^{2}} \frac{\eta^{\prime}\left(\frac{\left|x-\xi\left(s_{*}, t\right)\right|^{2}}{\varepsilon^{2}}\right)}{\varepsilon},
\end{aligned}
$$

and

$$
\xi_{s}\left(s_{*}, t\right) \cdot \nabla \psi(\xi(s, t))=\frac{o\left(\left(s-s_{*}\right)^{2}\right)}{\varepsilon^{2}} \frac{\eta^{\prime}\left(\frac{\left|x-\xi\left(s_{*}, t\right)\right|^{2}}{\varepsilon^{2}}\right)}{\varepsilon} .
$$

Plugging these estimates into (2.25) and noting that the boundary term there vanishes for $\varepsilon$ sufficiently small yields $0=c \gamma\left(s_{*}, t\right) b\left(s_{*}, t\right)+o(1)$ for some nonzero $c$, which shows that $b$ times $\gamma$ vanishes identically. Hence (2.25) reduces to

$$
0=\int_{s_{0}(t)}^{s_{1}(t)}\left[a \gamma \partial_{s} \psi(\xi(s, t))-\gamma_{t} \psi(\xi(s, t))\right] d s-\left.\left.\gamma \psi\right|_{s=s_{j}(t)} s_{j}^{\prime}(t)\right|_{j=0} ^{j=1} .
$$

In particular,

$$
0=\int_{s_{0}(t)}^{s_{1}(t)}\left[a \gamma \partial_{s} \psi(\xi(s, t))-\gamma_{t} \psi(\xi(s, t))\right] d s
$$

for every $\psi$ that vanishes at the endpoints $s_{j}(t)$. This implies that $a \gamma$ is differentiable with respect to $s$ for $s_{0}(t)<s<s_{1}(t)$ and $(a \gamma)_{s}=-\gamma_{t}$, i.e., the equation for $\gamma$ in (2.17) holds. Furthermore, since by construction $\xi_{t}+a \xi_{s}-\mathcal{U}=b \xi_{s}^{\perp}$, the fact that $b \gamma$ vanishes identically shows that the evolution equation for $\xi$ holds wherever $\gamma$ is nonzero. Since the expression $\xi_{t}+a \xi_{s}-\mathcal{U}$ is continuous it must then vanish except on open sets where $\gamma$ vanishes identically.

Finally, when at least one $s_{j}$ is finite, then integrating by parts in (2.26) now shows that

$$
0=\left.\left.\gamma \psi\right|_{s=s_{j}(t)}\left[s_{j}^{\prime}(t)-a\left(s_{j}(t), t\right)\right]\right|_{j=0} ^{j=1}
$$

and this implies (2.20) whether the $s_{j}$ are distinct or not. 
Remark. One of the issues that made the proof above long was our concern for including as many plausible examples of vortex sheet evolution as possible. For example, the Kaden spiral, periodic sheets and closed sheets all may be considered as smooth solutions.

\section{EQUiVALENCE OF WEAK FORMULATIONS}

This section is also divided into three subsections. In the first one we recall the concept of regular curves and G. David's result on Cauchy integrals on regular curves. In the second subsection we state and prove our main result, the criterion for the equivalence of Birkhoff-Rott and 2D Euler for vortex sheet evolution. In the final subsection we recall an example that establishes the sharpness of the criterion presented.

3.1. Regular curves. Let us begin by recalling the concept of regular curve. A rectifiable curve $\mathcal{C}$ is called regular if there exists a constant $A>0$ such that for any disk $D_{r}$ of radius $r>0$,

$$
\left|\mathcal{C} \cap D_{r}\right| \leq A r,
$$

where $\left|\mathcal{C} \cap D_{r}\right|$ represents the length of this segment of curve.

Let $\mathcal{C} \equiv\{\xi=\xi(s)\}$ be a rectifiable curve parametrized by arclength, with $s \in$ $\left(a_{0}, a_{1}\right)$. Let $\mathcal{U}_{*}$ be the maximal operator associated to $\mathcal{U}$, i.e.,

$$
\mathcal{U}_{*}[\gamma] \equiv \sup _{\varepsilon>0}\left|\int_{\left|\xi(s)-\xi\left(s^{\prime}\right)\right| \geq \varepsilon} K\left(\xi(s)-\xi\left(s^{\prime}\right)\right) \gamma\left(s^{\prime}\right) d s^{\prime}\right| .
$$

Theorem 3.1 (G. David, 6]). Suppose that $\mathcal{C}$ is a regular curve and let $1<p<\infty$. Then the maximal operator $\gamma \mapsto \mathcal{U}_{*}[\gamma]$ is a bounded sublinear operator from $L^{p}(d s)$ into $L^{p}(d s)$. Conversely, if there exists a continuous linear operator $\mathcal{U}: L^{2}(d s) \rightarrow$ $L^{2}(d s)$ such that, for any $\gamma \in C_{c}^{0}(d s)$ and for any $s_{0}$ such that $\xi\left(s_{0}\right)$ does not belong to $\xi(\operatorname{Supp}(\gamma))$, it holds that $\mathcal{U}[\xi ; \gamma]\left(s_{0}\right)=\int K\left(\xi\left(s_{0}\right)-\xi\left(s^{\prime}\right)\right) \gamma\left(s^{\prime}\right) d s^{\prime}$, then $\mathcal{C}$ is a regular curve.

Remark. (1) The result above was originally stated as a characterization of the rectifiable curves $\mathcal{C}$ in the complex plane such that the Cauchy integral defines a bounded operator from $L^{2}(\mathcal{C})$ to itself, namely, Theorem 2 in 6 . One may identify the integral in the definition of $\mathcal{U}_{*}$ with a Cauchy integral by introducing the usual identification of $\mathbb{R}^{2}$ with $\mathbb{C}$. In addition, we stated David's result in $L^{p}$, thereby incorporating the comment made immediately after the proof on page 174 of $[6]$.

(2) As remarked in [6], it follows that in the case of parametrization by arclength, $\mathcal{U}[\xi ; \gamma]$ is defined $d s$-almost everywhere. Furthermore, $\mathcal{U}$ defines a continuous linear operator from $L^{p}(d s)$ to itself.

3.2. The equivalence theorem. We are now ready to state and prove our main result.

Theorem 3.2. Let $\mathcal{C}_{t}=\left\{\xi=\xi(s, t) \mid s_{0}(t) \leq s \leq a_{1}(t)\right\}$ be a regular curve parametrized by arclength, $0 \leq t<T$. Assume that the constant $A$ in the definition of a regular curve, (3.1), may be chosen independently of $t$. Let

$$
\gamma \in L^{\infty}\left([0, T) ; L^{2}(d s) \cap L^{1}(d s)\right) .
$$


Then $(\gamma, \xi)$ is a weak solution of the Birkhoff-Rott equations with initial data $\left(\gamma_{0}, \xi_{0}\right)$ if and only if $\omega=\gamma \delta_{\mathcal{C}_{t}}$ is a weak solution of the weak vorticity formulation with initial data $\omega_{0}=\gamma_{0} \delta_{\mathcal{C}_{0}}, \mathcal{C}_{0}=\left\{\xi=\xi_{0}(s)\right\}$.

Before we proceed with the proof, let us emphasize that the only if portion of this result assumes that the weak solution of 2D Euler has the structure $\gamma \delta_{\mathcal{C}_{t}}$. Although existence of weak solutions with initial data of this kind has been established in certain cases, their structure is not known a priori.

Proof. There are two steps in this proof. The first step is to show that the identities (2.19) and (2.12) are the same under the hypothesis of this theorem. The second is to show that the regularity requirements in Definitions 2.2 and 2.1 are equivalent in this case as well.

We begin by showing that the identities (2.19) and (2.12), involving the test function $\varphi \in C_{c}^{\infty}\left([0, T) \times \mathbb{R}^{2}\right)$, are the same. First note that we need only consider the nonlinear term in each identity. We will show that, under our hypothesis, we have

$$
\begin{gathered}
\int_{0}^{T} \int_{s_{0}(t)}^{s_{1}(t)} \nabla \varphi(\xi(s, t), t) \cdot \mathcal{U}[\xi ; \gamma](s, t) \gamma(s, t) d s d t \\
=\int_{0}^{T} \int_{s_{0}(t)}^{s_{1}(t)} \int_{s_{0}(t)}^{s_{1}(t)} H_{\varphi}(\xi(r, t), \xi(s, t), t) \gamma(r, t) \gamma(s, t) d r d s d t
\end{gathered}
$$

for any test function $\varphi$. We start by recalling that

$$
\mathcal{U}[\xi ; \gamma]=\mathcal{U}[\xi ; \gamma](s, t)=\lim _{\varepsilon \rightarrow 0^{+}} \int_{|\xi(s, t)-\xi(r, t)| \geq \varepsilon} K(\xi(s, t)-\xi(r, t)) \gamma(r, t) d r .
$$

Fix $\varepsilon>0$. Denote by $\Delta_{\xi}(r, s, t):=\xi(s, t)-\xi(r, t)$. Note that

$$
\begin{aligned}
\int_{0}^{T} \int_{s_{0}(t)}^{s_{1}(t)} \nabla \varphi(\xi(s, t), t) \cdot\left(\int_{\left|\Delta_{\xi}(r, s, t)\right| \geq \varepsilon} K\left(\Delta_{\xi}(r, s, t)\right) \gamma(r, t) d r\right) \gamma(s, t) d s d t \\
(3.2) \quad=\int_{0}^{T} \int_{s_{0}(t)}^{s_{1}(t)} \int_{\left|\Delta_{\xi}(r, s, t)\right| \geq \varepsilon} \nabla \varphi(\xi(s, t), t) \cdot K\left(\Delta_{\xi}(r, s, t)\right) \gamma(r, t) \gamma(s, t) d r d s d t \\
=-\int_{0}^{T} \int_{s_{0}(t)}^{s_{1}(t)} \int_{\left|\Delta_{\xi}(r, s, t)\right| \geq \varepsilon} \nabla \varphi(\xi(r, t), t) \cdot K\left(\Delta_{\xi}(r, s, t)\right) \gamma(s, t) \gamma(r, t) d s d r d t,
\end{aligned}
$$

exchanging $s$ with $r$ and using the antisymmetry of the kernel $K$,

$$
=-\int_{0}^{T} \int_{s_{0}(t)}^{s_{1}(t)} \int_{\left|\Delta_{\xi}(r, s, t)\right| \geq \varepsilon} \nabla \varphi(\xi(r, t), t) \cdot K\left(\Delta_{\xi}(r, s, t)\right) \gamma(s, t) \gamma(r, t) d r d s d t
$$

using Fubini's theorem.

Hence, adding $1 / 2$ of (3.2) to $1 / 2$ of (3.3), we find that

$$
\begin{gathered}
\int_{0}^{T} \int_{s_{0}(t)}^{s_{1}(t)} \nabla \varphi(\xi(s, t), t) \cdot\left(\int_{\left|\Delta_{\xi}(r, s, t)\right| \geq \varepsilon} K\left(\Delta_{\xi}(r, s, t)\right) \gamma(r, t) d r\right) \gamma(s, t) d s d t \\
=\int_{0}^{T} \int_{s_{0}(t)}^{s_{1}(t)} \int_{\left|\Delta_{\xi}(r, s, t)\right| \geq \varepsilon} H_{\varphi}(\xi(r, t), \xi(s, t), t) \gamma(r, t) \gamma(s, t) d r d s d t .
\end{gathered}
$$


The right hand side of the identity above converges to the nonlinear term in (2.12) as $\varepsilon \rightarrow 0$, by the Dominated Convergence Theorem, since $H_{\varphi}$ is bounded and $\gamma$ was assumed to be integrable.

To establish that (2.19) and (2.12) are the same it remains to show that

$$
\begin{gathered}
\int_{0}^{T} \int_{s_{0}(t)}^{s_{1}(t)} \nabla \varphi(\xi(s, t), t) \cdot \mathcal{U}[\xi ; \gamma](s, t) \gamma(s, t) d s d t \\
=\lim _{\varepsilon \rightarrow 0} \int_{0}^{T} \int_{s_{0}(t)}^{s_{1}(t)} \nabla \varphi(\xi(s, t), t) \cdot\left(\int_{\left|\Delta_{\xi}(r, s, t)\right| \geq \varepsilon} K\left(\Delta_{\xi}(r, s, t)\right) \gamma(r, t) d r\right) \gamma(s, t) d s d t .
\end{gathered}
$$

We first note that

$$
\left|\int_{\left|\Delta_{\xi}(r, s, t)\right| \geq \varepsilon} K\left(\Delta_{\xi}(r, s, t)\right) \gamma(r, t) d r\right| \leq \mathcal{U}_{*}[\gamma],
$$

where $\mathcal{U}_{*}$ is the maximal operator associated to $\mathcal{U}[\cdot, \xi]$ introduced in Section 2 . Since $\gamma \in L^{\infty}\left((0, T) ; L^{2}(d s)\right)$, it follows from Theorem 3.1 that, $\mathcal{U}_{*}[\gamma] \in L^{2}(d s)$, for almost all time. Furthermore, since $\mathcal{U}_{*}$ is a bounded sublinear operator from $L^{2}$ to itself, we have in fact that $\mathcal{U}_{*}[\gamma] \in L^{\infty}\left((0, T) ; L^{2}(d s)\right)$. Therefore $\nabla \varphi \cdot \mathcal{U}_{*}[\gamma] \gamma \in$ $L^{\infty}\left((0, T) ; L^{1}(d s)\right)$. The desired identity follows by dominated convergence.

The second step in this proof is to examine regularity conditions in Definition 2.2 and Definition 2.1. First note that any density $\gamma$ and curve $\xi$ satisfying the hypothesis of this theorem will satisfy the regularity requirements of Definition 2.2. Indeed, by Theorem 3.1, $\mathcal{U}[\xi ; \gamma] \in L^{\infty}\left((0, T) ; L^{2}(d s)\right)$ so that $\gamma \mathcal{U}[\xi ; \gamma] \in$ $L^{\infty}\left((0, T) ; L^{1}(d s)\right)$. On the other hand, the fact that $\gamma \in L^{\infty}\left((0, T) ; L^{1}(d s)\right)$ implies that $\omega \in L^{\infty}\left((0, T) ; \mathcal{B M}_{c}\right)$.

Under the conditions of the theorem, the vorticity also automatically satisfies the condition

$$
\omega \in L^{\infty}\left((0, T) ; H_{l o c}^{-1}\left(\mathbb{R}^{2}\right)\right)
$$

needed to obtain the equivalence of the weak vorticity formulation with the definitive weak velocity formulation. In addition, $\omega$ also satisfies the condition

$$
\omega \in \operatorname{Lip}\left((0, T) ; H_{l o c}^{-L}\left(\mathbb{R}^{2}\right)\right) \text { for some } L>1
$$

that ensures the compactness of bounded sequences of solutions.

To prove (3.4), we begin by observing that, given any disk $D_{r}$ of radius $r>0$ in the plane, we have

$$
\left|\omega\left(D_{r}\right)\right|=\left|\int_{\mathcal{C}_{t} \cap D_{r}} \gamma d s\right| \leq\|\gamma\|_{L^{2}}^{1 / 2}(A r)^{1 / 2} \leq C r^{1 / 2},
$$

by Cauchy-Schwarz and using the definition of regular curve, for some constant $C>0$ independent of time. Hence the vorticity $\omega$ belongs to $L^{\infty}\left((0, T) ; \widetilde{M}^{4 / 3}\left(\mathbb{R}^{2}\right)\right)$, where $\widetilde{M}^{4 / 3}$ is a Morrey space of measures [22. It was shown in [22], Theorem 4.3, that for any $p>n / 2, \widetilde{M}^{p}\left(\mathbb{R}^{n}\right) \cap \mathcal{B} \mathcal{M}_{\text {loc }}\left(\mathbb{R}^{n}\right)$ is (compactly) contained in $H_{\text {loc }}^{-1}\left(\mathbb{R}^{n}\right)$.

Now consider (3.5). We have already shown that, since $(\gamma, \xi)$ was assumed to satisfy Definition 2.2, then $(\gamma, \xi)$ satisfies identity (2.12) for any test function 
$\varphi \in C_{c}^{\infty}\left([0, T) \times \mathbb{R}^{2}\right)$. In particular, if $\varphi \in C_{c}^{\infty}\left((0, T) \times \mathbb{R}^{2}\right)$, then

$$
\begin{gathered}
\int_{0}^{T} \int_{s_{0}(t)}^{s_{1}(t)} \varphi_{t}(\xi(s, t), t) \gamma(s, t) d s d t \\
=-\int_{0}^{T} \int_{s_{0}(t)}^{s_{1}(t)} \int_{s_{0}(t)}^{s_{1}(t)} H_{\varphi}(\xi(r, t), \xi(s, t), t) \gamma(r, t) \gamma(s, t) d r d s d t .
\end{gathered}
$$

Observe that $\left\|H_{\varphi}(\cdot, \cdot, t)\right\|_{L^{\infty}\left(\mathbb{R}^{4}\right)} \leq\|\varphi(\cdot, t)\|_{W^{2, \infty}\left(\mathbb{R}^{2}\right)}$. Note also that

$$
\int_{0}^{T} \int_{s_{0}(t)}^{s_{1}(t)} \varphi_{t}(\xi(s, t), t) \gamma(s, t) d s d t=\int_{0}^{T} \int_{\mathbb{R}^{2}} \varphi_{t} \omega(x, t) d x d t
$$

In view of these observations, together with the hypothesis on $\gamma$, we deduce that

$$
\left|\int_{0}^{T} \int_{\mathbb{R}^{2}} \varphi_{t} \omega d x d t\right| \leq C\|\varphi\|_{L^{1}\left((0, T) ; W^{2, \infty}\left(\mathbb{R}^{2}\right)\right)}
$$

for any test function in $C_{c}^{\infty}\left((0, T) \times \mathbb{R}^{2}\right)$,

$$
\leq C\|\varphi\|_{L^{1}\left((0, T) ; H^{4}\left(\mathbb{R}^{2}\right)\right)}
$$

by the Morrey inequality. By duality this implies that the distribution $\omega_{t} \in$ $L^{\infty}\left((0, T) ; H^{-4}\left(\mathbb{R}^{2}\right)\right)$, which in turn gives (3.5) with $L=4$.

Remark. The hypothesis that $\gamma \in L^{\infty}\left((0, T) ; L^{1}(d s)\right)$ is redundant, as it is explicitly present in Definition 2.2 and implicitly present in Definition 2.1. We included this hypothesis in the statement of the theorem merely for the sake of clarity.

3.3. Sharpness. Next we will observe that the condition $\gamma \in L^{2}(d s)$, needed above to prove the equivalence of the weak formulations of $2 \mathrm{D}$ Euler and Birkhoff-Rott, is sharp. To see this, we recall the classical example of the Prandtl-Munk vortex sheet, see [30, also known as the elliptically loaded wing; see [18]. We consider flow generated by the initial vortex sheet given by

$$
\mathcal{C}_{0}=\left\{\xi_{0}(s)=(s, 0),-1 \leq s \leq 1\right\} \text { and } \gamma_{0}(s)=\frac{s}{\sqrt{1-s^{2}}}
$$

By complex variable methods it can be shown that the velocity $\mathcal{U}_{0}\left[\xi_{0} ; \gamma_{0}\right](s) \equiv$ $(0,-1 / 2)$; see [36], section 6.2. This is pointwise true for $-1<s<1$, but unclear at $s= \pm 1$, since the flow velocity is infinite near the tips of the sheet.

The fact that the arithmetic mean $\mathcal{U}$ is constant suggests that $\xi(s, t)=(s,-t / 2)$, $\gamma(s, t)=\gamma_{0}(s)$ describes the evolution of this vortex sheet. In fact, $(\gamma, \xi)$ is indeed a weak solution of the Birkhoff-Rott equations with initial data $\left(\gamma_{0}, \xi_{0}\right)$ in the sense of Definition 2.2. The verification of this fact is straightforward. On the other hand, it was shown in 21] that $\omega=\gamma \delta_{\mathcal{C}_{t}}$ is not a weak solution of the incompressible 2D Euler equations with initial data $\gamma_{0} \delta_{\mathcal{C}_{0}}$ in the sense of Definition 2.1. We observe that $\gamma \in L^{1}(d s) \cap L^{p}(d s)$ for all $p<2$ but not for $p=2$, which shows the sharpness of the $L^{2}$ condition on $\gamma$ in Theorem 3.2 . 


\section{FinAL REMARKS}

Recently, G. Lebeau proved that any solution of the Birkhoff-Rott system consisting of a closed vortex sheet which is $C^{1, \alpha}$ at a time $t_{0}$ is real-analytic for $t \neq t_{0}$; see [19]. This is a consequence of the elliptic nature of the Birkhoff-Rott equations. In [40], Sijue Wu announced an improvement of Lebeau's result, in which the conclusion is mantained if the vortex sheet is required to be a chord-arc curve and satisfies an additional technical condition. Thus, one might conclude that there are no irregular solutions of Birkhoff-Rott and wonder whether there is any reason to formulate a general theory of equivalence of Euler and weak Birkhoff-Rott. In

that regard, we observe that the Prandtl-Munk vortex sheet is an example of an irregular solution of Birkhoff-Rott which is not covered by Lebeau's work because it is not a closed sheet, so that the context behind our result is not entirely empty. We also note that the class of regular curves is broader than chord-arc, hence solutions which are regular curves but which are not real analytic are plausible. In particular, the logarithmic spirals of Prandtl and Kambe fit into our discussion.

In Theorem 3.2 on the equivalence between the Euler and Birkhoff-Rott descriptions of vortex sheet motion we restrict our attention solely to regular curves. This hypothesis is motivated by hindsight, since, David's result includes the fact that the Cauchy integral on a rectifiable curve is a bounded operator on $L^{2}$ only if the curve is regular, as stated in Theorem 3.1, Although the Birkhoff-Rott equations might make sense even if the principal-value integral does not give rise to a bounded operator in $L^{2}$, this becomes a substantial complication. However, the hypothesis that the sheet be regular is a big limitation in our work, since the conjectured behavior past singularity formation for periodic sheets is the development of infinite length algebraic spirals, which are hence not regular.

Let us recall that the existence of vortex sheet evolution, at least with distinguished sign density, has been established from the implicit approach. Why then would we still want to solve Birkhoff-Rott? The main motivation is that the Birkhoff-Rott equations carry a much more precise description of the flow than the weak formulation of 2D Euler. In particular, the Birkhoff-Rott equations assume a priori that the evolution of a vortex sheet retains a curve-like structure, whereas the structure of the weak solutions given, for instance by Delort's Theorem, is not known. One interesting open problem is to prove that the support of a solution given by Delort's Theorem, with a smooth vortex sheet as initial data, has Lebesgue measure zero (better yet, Hausdorff dimension less than 2) at almost every time.

Another interesting question is to find a meaningful way in which curves which are not regular may be regarded as solutions of Birkhoff-Rott. At this point it would be helpful to have examples of physically meaningful vortex sheets having infinite length algebraic spirals, something which is not available at present. In this context it is worth mentioning a well-known open problem in the field, that of establishing existence of the Kaden spiral, or, more generally, whether there exist self-similar, algebraic spiral solutions of the Birkhoff-Rott equations.

\section{ACKNOWLEDGMENTS}

The authors wish to thank Max Souza and Sijue Wu for helpful discussions. The first author's research was supported in part by CNPq grant \# 300.962/91-6. The second author's research was supported in part by CNPq grant \# 300.158/93-9. 


\section{REFERENCES}

[1] G. K. Batchelor. An introduction to fluid dynamics. Cambridge Mathematical Library. Cambridge University Press, Cambridge, 1999. MR1744638 (2000j:76001)

[2] D. Benedetto and M. Pulvirenti. From vortex layers to vortex sheets. SIAM J. Appl. Math., 52(4):1041-1056, 1992. MR 1174045 (93g:76027)

[3] G. Birkhoff. Helmholtz and Taylor instability. Proc. Sympos. Appl. Math., Vol. XIII, pages 55-76. American Mathematical Society, Providence, R.I., 1962. MR0137423 (25:875)

[4] R. Caflisch and O. Orellana. Long time existence for a slightly perturbed vortex sheet. Comm. Pure Appl. Math., 39(6):807-838, 1986. MR0859274 (87m:76018)

[5] R. Caflisch and O. Orellana. Singular solutions and ill-posedness for the evolution of vortex sheets. SIAM J. Math. Anal., 20(2):293-307, 1989. MR0982661 (90d:76020)

[6] G. David. Opérateurs intégraux singuliers sur certaines courbes du plan complexe. Ann. Sci. École Norm. Sup. (4), 17(1):157-189, 1984. MR0744071 (85k:42026)

[7] J.-M. Delort. Existence de nappes de tourbillon en dimension deux. J. Amer. Math. Soc., 4(3):553-586, 1991. MR1102579 (92f:76019)

[8] R. DiPerna and A. Majda. Concentrations in regularizations for 2-D incompressible flow. Comm. Pure Appl. Math., 40(3):301-345, 1987. MR0882068 (88e:35149)

[9] R. DiPerna and A. Majda. Oscillations and concentrations in weak solutions of the incompressible fluid equations. Comm. Math. Phys., 108(4):667-689, 1987. MR.0877643(88a:35187)

[10] R. DiPerna and A. Majda. Reduced Hausdorff dimension and concentration-cancellation for two-dimensional incompressible flow. J. Amer. Math. Soc., 1(1):59-95, 1988. MR0924702 (89e:35126)

[11] J. Duchon and R. Robert. Global vortex sheet solutions of Euler equations in the plane. J. Differential Equations, 73(2):215-224, 1988. MR0943940 (89h:35262)

[12] L. C. Evans. Weak convergence methods for nonlinear partial differential equations. CBMS Lecture Notes v. 74, American Math. Soc., Providence, 1990. MR.1034481 (91a:35009)

[13] L. C. Evans and S. Müller. Hardy spaces and the two-dimensional Euler equations with nonnegative vorticity. J. Amer. Math. Soc., 7(1):199-219, 1994. MR1220787 (94h:35205)

[14] H. Kaden. Aufwicklung einer unstabilen unstetigkeitsfläsche. Ing. Arch., 2:140-168, 1931.

[15] T. Kambe. Spiral vortex solution of Birkhoff-Rott equation. Phys. D, 37(1-3):463-473, 1989. MR1024399 (90k:76031)

[16] R. Krasny. Desingularization of periodic vortex sheet roll-up. J. Comput. Phys., 65:292-313, 1986.

[17] R. Krasny. A study of singularity formation in a vortex sheet by the point-vortex approximation. J. Fluid Mech., 167:65-93, 1986. MR0851670 (87g:76028)

[18] R. Krasny. Computation of vortex sheet roll-up in the Trefftz plane. J. Fluid Mech., 184:123$155,1987$.

[19] G. Lebeau. Régularité du problème de Kelvin-Helmholtz pour l'équation d'Euler 2d. ESAIM Control Optim. Calc. Var., 8:801-825 (electronic), 2002. MR1932974 (2004a:76011)

[20] J.-G. Liu and Z. Xin. Convergence of vortex methods for weak solutions to the 2-D Euler equations with vortex sheet data. Comm. Pure Appl. Math., 48(6):611-628, 1995. MR.1338471 (96c:76016)

[21] M. C. Lopes Filho, H. J. Nussenzveig Lopes, and M. O. Souza. On the equation satisfied by a steady Prandtl-Munk vortex sheet. Commun. Math. Sci., 1(1):68-73, 2003. MR1979844 (2004c:76029)

[22] M. C. Lopes Filho, H. J. Nussenzveig Lopes, and E. Tadmor. Approximate solutions of the incompressible Euler equations with no concentrations. Ann. Inst. H. Poincaré Anal. Non Linéaire, 17(3):371-412, 2000. MR1771138 (2001h:35152)

[23] M. C. Lopes Filho, H. J. Nussenzveig Lopes, and Z. Xin. Existence of vortex sheets with reflection symmetry in two space dimensions. Arch. Ration. Mech. Anal., 158(3):235-257, 2001. MR1842346 (2002e:35197)

[24] A. Majda. The interaction of nonlinear analysis and modern applied mathematics. Proceedings of the International Congress of Mathematicians, Vol. I, II (Kyoto, 1990), pages 175-191, Tokyo, 1991. Math. Soc. Japan. MR1159212 (93g:35003a)

[25] A. Majda. Remarks on weak solutions for vortex sheets with a distinguished sign. Indiana Univ. Math. J., 42(3):921-939, 1993. MR1254126 (94k:35236) 
[26] A. Majda. and A. Bertozzi. Vorticity and incompressible flow, Cambridge Texts in Applied Mathematics, Cambridge Univ. Press, Cambridge, 2002. MR.1867882 (2003a:76002)

[27] C. Marchioro and M. Pulvirenti. Mathematical theory of incompressible nonviscous fluids, volume 96 of Applied Mathematical Sciences. Springer-Verlag, New York, 1994. MR 1245492 (94k:76001)

[28] D. Meiron, G. Baker, and S. Orszag. Analytic structure of vortex sheet dynamics. I. KelvinHelmholtz instability. J. Fluid Mech., 114:283-298, 1982. MR0647268 (83c:76017)

[29] D. W. Moore. The spontaneous appearance of a singularity in the shape of an evolving vortex sheet. Proc. Roy. Soc. London Ser. A, 365(1720):105-119, 1979. MR0527594 (80b:76006)

[30] M. Munk. Isoperimetrische Aufgaben aus der Theorie des Fluges. Inaug.-dissertation, Göttingen, 1919.

[31] H. J. Nussenzveig Lopes. A refined estimate of the size of concentration sets for 2D incompressible inviscid flow. Indiana Univ. Math. J., 46(1):165-182, 1997. MR 1462801(98h:35191)

[32] S. Osher and R. Fedkiw. Level set methods and dynamic implicit surfaces, volume 153 of Applied Mathematical Sciences. Springer-Verlag, New York, 2003. MR 1939127|(2003j:65002)

[33] L. Prandtl. Uber die Entstehung von Wirbeln in der idealen Flussigkeit, mit Anwendung auf die Tragflugeltheorie und andere Aufgaben, in Von Kármán and Levi-Civita (eds.) Vorträge aus dem Gebiete der Hydro- und Aerodynamik, Springer, Berlin, 1922.

[34] D. I. Pullin, J. D. Buntine, and P. G. Saffman. On the spectrum of a stretched spiral vortex. Phys. Fluids, 6(9):3010-3027, 1994. MR.1290064 (95f:76047)

[35] N. Rott. Diffrraction of a weak shock with vortex generation. J. Fluid Mech., 1:111-128, 1956. MR0082327 (18:534e)

[36] P. G. Saffman. Vortex dynamics. Cambridge Monographs on Mechanics and Applied Mathematics. Cambridge University Press, New York, 1992. MR.1217252 (94c:76015)

[37] S. Schochet. The weak vorticity formulation of the 2-D Euler equations and concentrationcancellation. Comm. Partial Differential Equations, 20(5-6):1077-1104, 1995. MR1326916 (96h:35155)

[38] S. Schochet. The point-vortex method for periodic weak solutions of the 2-D Euler equations. Comm. Pure Appl. Math., 49(9):911-965, 1996. MR1399201 (97e:35137)

[39] C. Sulem, P.-L. Sulem, C. Bardos, and U. Frisch. Finite time analyticity for the two- and three-dimensional Kelvin-Helmholtz instability. Comm. Math. Phys., 80(4):485-516, 1981. MR0628507 (83d:76012)

[40] S. Wu. Recent progress in mathematical analysis of vortex sheets. In Proceedings of the International Congress of Mathematicians, Vol. III (Beijing, 2002), pages 233-242, Beijing, 2002. Higher Ed. Press. MR 1957535 (2003m:76015)

[41] Y. Zheng. Concentration-cancellation for the velocity fields in two-dimensional incompressible fluid flows. Comm. Math. Phys., 135(3):581-594, 1991. MR1091579(92e:35131)

Departamento de Matemática, imeCC-Unicamp, Cx. Postal 6065, Campinas SP 13081-970, BRAZIL

E-mail address: mlopes@ime.unicamp.br

Departamento de Matemática, imeCC-Unicamp, Cx. Postal 6065, Campinas SP 13081-970, BRAZIL

E-mail address: hlopes@ime.unicamp.br

School of Mathematical Sciences, Tel Aviv University, Ramat Aviv, Tel Aviv 69978 ISRAEL

E-mail address: schochet@post.tau.ac.il 